\title{
Challenges in the clinical development pathway for triple and multiple drug combinations in the treatment of uncomplicated falciparum malaria
}

\author{
Quique Bassat 1,2,3,4, , Oumou Maïga-Ascofaré6,7,8 , Jürgen May ${ }^{7,8}$, Jerôme Clain ${ }^{9}$, Ghyslain Mombo-Ngoma ${ }^{7,8,10}$, \\ Mirjam Groger ${ }^{7,8}$, Ayôla A. Adegnika ${ }^{10,11}$, Jean-Claude Dejon Agobé ${ }^{10}$, Abdoulaye Djimde ${ }^{12}$, \\ Johannes Mischlinger ${ }^{7,8}$ and Michael Ramharter ${ }^{7,8,10^{*}}$ (D) on behalf of ASAAP and Multimal Consortia
}

\begin{abstract}
The addition of a third anti-malarial drug matching the pharmacokinetic characteristics of the slowly eliminated partner drug in artemisinin-based combination therapy (ACT) has been proposed as new therapeutic paradigm for the treatment of uncomplicated falciparum malaria. These triple artemisinin-based combination therapy (TACT) should in theory more effectively prevent the development and spread of multidrug resistance than current ACT. Several clinical trials evaluating TACT—or other multidrug anti-malarial combination therapy (MDACT) — have been reported and more are underway. From a regulatory perspective, these clinical development programmes face a strategic dilemma: pivotal clinical trials evaluating TACT are designed to test for non-inferiority of efficacy compared to standard ACT as primary endpoint. While meeting the endpoint of non-inferior efficacy, TACT are consistently associated with a slightly higher frequency of adverse drug reactions than currently used ACT. Moreover, the prevention of the selection of specific drug resistance - one of the main reasons for TACT development-is beyond the scope of even large-scale clinical trials. This raises important questions: if equal efficacy is combined with poorer tolerability, how can then the actual benefit of these drug combinations be demonstrated? How should clinical development plans be conceived to provide objective evidence for or against an improved management of patients and effective prevention of antimalarial drug resistance by TACT? What are the objective criteria to ultimately convince regulators to approve these new products? In this Opinion paper, the authors discuss the challenges for the clinical development of triple and multidrug anti-malarial combination therapies and the hard choices that need to be taken in the further clinical evaluation and future implementation of this new treatment paradigm.
\end{abstract}

Keywords: Malaria, Artemisinin-based combination therapy, Falciparum, Regulatory pathway

\section{Background}

Since the Second World War monotherapy constituted the universal treatment paradigm for falciparum malaria [1]. When drug resistance emerged in a geographical

\footnotetext{
*Correspondence: ramharter@bnitm.de

${ }^{7}$ Bernhard Nocht Institute for Tropical Medicine, Hamburg, Germany

Full list of author information is available at the end of the article
}

region (most frequently initially in South-East Asia), a new anti-malarial drug was evaluated in clinical trials against a failing first-line therapy demonstrating superior efficacy and ultimately replacing it as standard treatment for uncomplicated malaria [1-3]. This process occurred in similar ways for the major anti-malarials of the twentieth century-chloroquine, sulfadoxine-pyrimethamine, and mefloquine-until finally no replacement drug was original author(s) and the source, provide a link to the Creative Commons licence, and indicate if changes were made. The images or other third party material in this article are included in the article's Creative Commons licence, unless indicated otherwise in a credit line to the material. If material is not included in the article's Creative Commons licence and your intended use is not permitted by statutory regulation or exceeds the permitted use, you will need to obtain permission directly from the copyright holder. To view a copy of this licence, visit http://creativecommons.org/licenses/by/4.0/. The Creative Commons Public Domain Dedication waiver (http://creativeco mmons.org/publicdomain/zero/1.0/) applies to the data made available in this article, unless otherwise stated in a credit line to the data. 
readily available for clinical testing $[4,5]$. In parallel, the spread of these drug resistant Plasmodium falciparum isolates throughout sub-Saharan Africa was paralleled by a dramatic increase in malaria-related mortality, highlighting the impact of drug resistance from a public health perspective [6].

To address this vicious cycle of drug development and emergence of drug resistance, and mimicking what had been historically proposed for other infectious diseases, such as tuberculosis (TB) or HIV, a new treatment paradigm emerged at the turn of the century by proposing artemisinin-based combination therapy (ACT) as standard of care for uncomplicated malaria [4]. ACT demonstrated higher cure rates than the hitherto failing first-line drug mefloquine. Over the following decade (and until today in most of the malaria endemic world outside of the Greater Mekong Region) ACT showed unprecedentedly high cure rates, favourable tolerability and safety and contributed importantly to the subsequent reduction of the global malaria burden.

\section{Rationale for evaluation of triple artemisinin-based combinations therapy (TACT) and multidrug anti-malarial combination therapy (MDACT)}

Whereas it was initially hoped that ACT will prevent the further emergence of drug resistance due to the combination of two independently acting drugs, this assumption was unfortunately not substantiated [7]. Over the past two decades the emergence of $P$. falciparum strains insufficiently responsive to conventional ACT in the Greater Mekong Region has led to a challenging public health situation for this region [8-10]. The use of ACT is associated with unacceptably low cure rates in some regions and the registration of drugs belonging to novel classes of anti-malarials will still require several years [911]. To overcome this situation triple artemisinin-based combination therapy (TACT) has been proposed as new treatment paradigm to bridge this period of time until new drugs become licensed ensuring high cure rates for multidrug resistant falciparum malaria [11, 12].

The therapeutic concept of TACT is-at least in partmotivated by two factors. Firstly, insights into the molecular mechanism of anti-malarial drug resistance indicates counter-selection of drug resistance by piperaquine and mefloquine-two of the most widely used partner drugs. Secondly-and more importantly for the concept of other TACT and MDACT-the rationale of combining three (or more) anti-malarial drugs relies in more general terms on the conceptual framework of mutual protection of the long acting partner drugs sharing similar pharmacokinetic characteristics from the selection of multiple random drug resistance mutations [12] (Table 1).
The concept of TACT has been born out of necessity by failing artemisinin-based combinations in the Greater Mekong Region, while new drugs are not yet available on the market. However, it is understood that the full potential of TACT to prevent drug resistance is its use in settings where drug resistance against any of the partner drugs has not yet emerged as adding a drug to a failing regimen may accelerate the development of parasites resistant to all drugs combined in the anti-malarial combination [12]. Today the Greater Mekong Region harbours only low absolute numbers of falciparum infections, whereas sub-Saharan Africa is home to $94 \%$ of the global malaria burden [13]. If TACT and MDACT are to live up to their full potential, it is therefore understood that this new therapeutic concept needs to be introduced in sub-Saharan Africa before artemisinin-based combinations start to fail there. Given recent reports about kelch13 mutants associated with delayed parasite clearance times, the window of opportunity may be rather short on the African continent [14].

\section{Considerations on the efficacy outcomes of triple and multiple drug combinations for the treatment of malaria}

In contrast to the historic situation where a new antimalarial monotherapy was tested in a clinical trial aiming to demonstrate superior efficacy compared to a failing first-line drug, this situation changed with the development of the first artemisinin-based combinations. Subsequent combinations were then largely evaluated in clinical phase III trials aiming for the proof of non-inferiority of PCR corrected efficacy compared to the standard ACT. The clinical development of TACT now leads again to a paradigm shift as TACT constitute a new treatment paradigm that is developed with the intention to have high cure rates in settings of multidrug resistance, arguing for the requirement for superiority trials compared to standard ACT. However, to allow for TACT's full potential in the prevention of drug resistance, the clinical development of TACT would ideally take place in settings where ACT remains efficacious. This is then a challenging situation from the perspective of a clinical development plan as TACT can so only be tested for non-inferiority of efficacy compared to standard ACT as primary endpoint. Non-inferiority of PCR corrected adequate clinical and parasitological response becomes the main efficacy outcome due to the continued high cure rates $>95 \%$ of the comparator ACT in the absence of drug resistance against artemisinins and their partner drugs $[11,15]$.

Conclusive evidence from clinical trials evaluating TACT in regions where ACT is currently failing is also not a feasible option due to the low number of malaria 


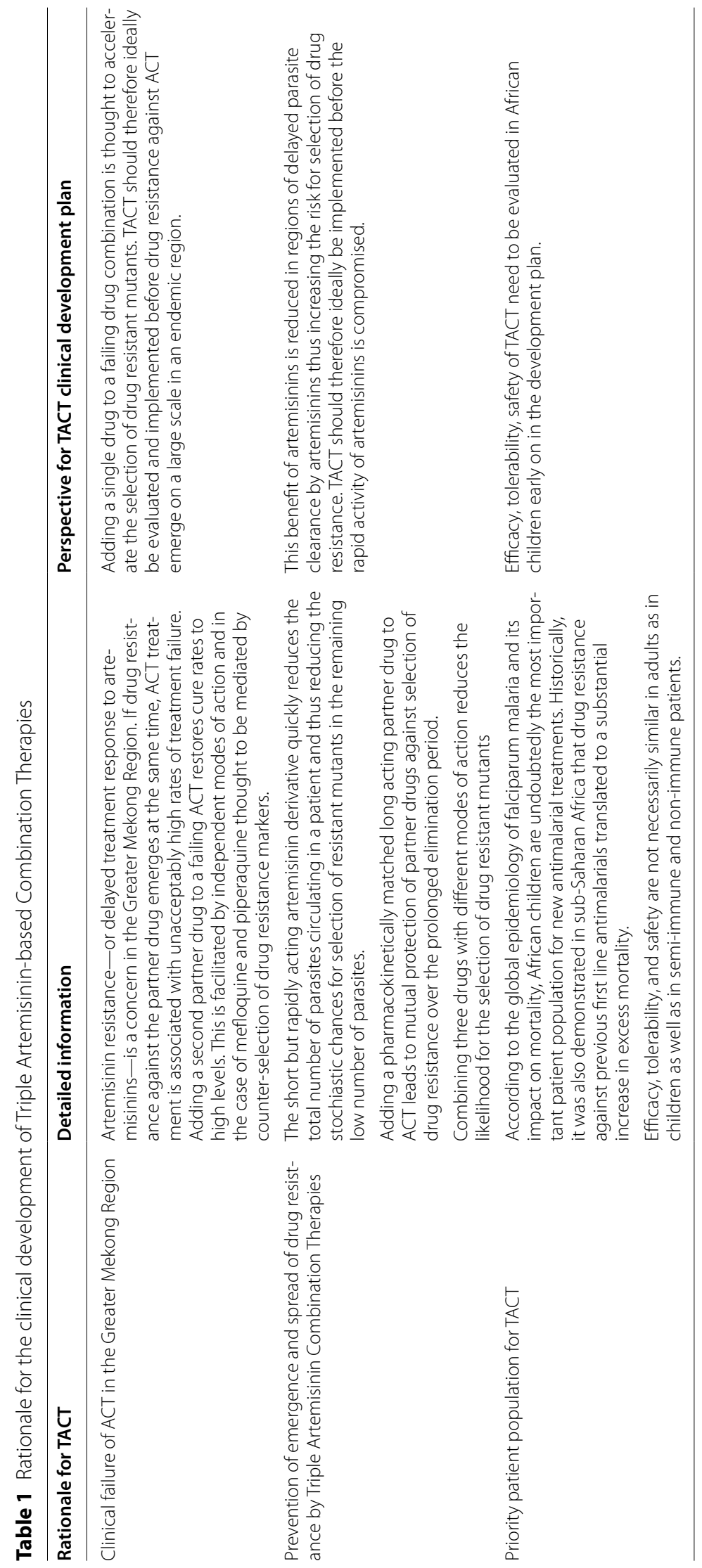


cases in the Greater Mekong Region. This low number precludes the recruitment of a sufficient number of patients to demonstrate superiority in efficacy in large clinical phase III trials. This fact was demonstrated by the first large clinical trial assessing TACT, which ultimately chose to enlarge the initial clinical trial network to include recruitment at sites outside of the Greater Mekong Region and thus switching from a superiority to a non-inferiority endpoint for efficacy analysis for most of the recruitment centres [11]. Whereas this change in recruitment strategy may be seen as a weakness for the primary efficacy analysis, it allowed at the same time to evaluate TACT in the most important patient population of interest - African children.

In summary, even if TACT is evaluated in regions of high prevalence of multidrug resistance, the low incidence of malaria in these regions precludes recruitment of sufficient numbers of patients for large clinical phase III trials. At the same time the theoretical rationale for TACT favours to primarily evaluate these therapeutic regimens in regions with high susceptibility to ACT and in African children. New TACT, therefore, needs to be evaluated in paediatric patients in sub-Saharan Africa to allow their safe use in this most important patient population. This necessary choice comes with the consequence of having to accept non-inferiority of efficacy as the primary pharmacodynamics endpoint.

\section{Considerations on the tolerability and safety outcomes of triple and multiple drug combinations for the treatment of malaria}

Combining two or more drugs in a therapeutic regimen is inevitably leading to a higher number of adverse drug reactions compared to monotherapy as each compound comes with its own off-target effects. Clinical development programmes for TACT, therefore, need to be carefully designed to assess the frequency and severity of adverse events to allow for an informed appreciation of the side effect profile of a new TACT regimen besides pharmacokinetic drug-drug interactions. Most common tolerability findings may include rather unspecific findings of asthenia, or gastrointestinal disturbances including abdominal pain, nausea and vomiting or allergic drug reactions $[11,15]$. As an unbiased quantitative estimation of tolerability is of high importance for the riskbenefit analysis of these therapeutic regimens compared to standard ACT, blinding of study patients and investigators to the treatment allocation is of high importance, even more so than in conventional drug development programmes.

At the same time a diligent safety assessment is of high importance as the combination of more than one drug may lead to anticipated and indeed unanticipated safety signals. The most common safety concerns involve drug induced hepatic injury and cardiac toxicity but other safety signals including renal, pancreatic, haematological findings as well as neurological side effects are of importance, too. It is recommended that these potential safety signals are investigated proactively and diligently by clinical assessments and diagnostic procedures as safety assurance arguably is of even higher importance in combination regimens than in monotherapy. Frequent safety signals may be detected in clinical phase I, II, and III trials. Rare, but potentially life-threatening safety events, may however only be reliably detected and their frequency estimated in large phase IV trials or post-marketing surveillance. This is a challenge that requires adequate attention to ensure safety assurance of new TACT in sub-Sahara Africa. In summary, currently reported clinical trials indicate that TACT will most likely result in slightly worse tolerability, while still demonstrating good safety profile [11]. Safety needs to be addressed proactively in clinical trials and in post-marketing registration studies to ensure maximum reassurance for the safe use of TACT.

\section{Ancillary endpoints in TACT clinical development programmes}

Clinical development plans of TACT should include key outcomes supporting the rationale for the use of TACT. This includes the assessment of molecular markers for drug resistance at recruitment of patients into clinical trials as well as reassessment in case of re-appearance of parasitaemia or re-treatment. This analysis may allow for a better understanding of potential selection of drug resistant mutants. TACT may also have a substantial impact on the onward transmission of malaria by targeting the sexual developmental stages of Plasmodium, blocking the sexual cycle in the mosquito or by a direct lethal effects on the vector [16-18].

Clinical development plans should, therefore, include these important aspects to quantify any of these features of anti-malarial treatment. The prevention of the development of drug resistance and its spread by the vector is one of the key rationales for TACT, which is why direct evidence from these clinical trials becomes critical.

At the same time overenthusiasm in these ancillary analyses needs to be cautioned. It is highly unlikely that molecular analysis of drug resistance markers from a single clinical trial will lead to conclusive evidence for the prevention of drug resistance by TACT. This is due to the fact that only a small number of patients (usually $<10 \%$ of recruited participants) will experience re-appearing parasitaemia in high transmission regions due to the 
highly efficacious therapeutic regimens [11]. Contrary to other viral and bacterial infectious diseases, such as HIV and $\mathrm{TB}$, selection of de novo drug resistance is overall a rare event in the eukaryote $P$. falciparum, probably also due to the rapid elimination of malaria parasites as compared to mycobacteria or HIV. Contrary to early HIV treatment trials indicating selection of drug resistance in virtually all individual patients, selection of anti-malarial drug resistance constitutes rare events potentially occurring only a few times within a decade in an entire population living under massive anti-malarial drug pressure in an endemic region $[19,20]$. This is, therefore, not an event that can be reliably assessed in vivo in individual clinical trials or clinical development programmes. However, once a drug resistant mutant has emerged, it may spread much more quickly in malaria compared to HIV or TB due to malaria's higher reproductive number [21]. Reporting of resistance markers is, therefore, important for future metanalytic approaches and mathematic modelling to assess the true preventive effect of triple and multiple drug combinations against the development and spread of drug resistant P. falciparum parasites.

In summary, the evaluation of ancillary endpoints such as the selection of molecular resistance markers and transmission blocking potential are of high importance and shall be incorporated in trials evaluating triple artemisinin-based combinations. At the same time, it is unlikely that single clinical trials and clinical trial programmes will lead to conclusive evidence in these key aspects of TACT use. The ultimate aspiration of TACT to prevent the development of drug resistance may only be appreciated in ecological studies, with in vivo and molecular drug resistance data available once this treatment concept has been implemented in large geographical settings for a sufficient period of time, or by pooling and meta-analysing the information generated through several different trials.

\section{TACT: a difficult perspective for funders, regulators, and manufacturers}

As elaborated above, in the current situation triple artemisinin-based combinations are most likely to demonstrate non-inferior efficacy, slightly worse tolerability, and inconclusive evidence for their potential to prevent the development and spread of drug resistance while indicating the absence of frequent safety issues in currently ongoing clinical development programs. This constitutes a seemingly unattractive combination of key characteristics for a therapeutic product and thus potentially putting in question their further clinical development. Given that this also comes with inevitably increased costs of tripleand multidrug combinations, and the need for the development of new fixed dose combination formulations compared to conventional artemisinin-based combinations by adding another drug to the existing combination, this situation is indeed not the default case for funders, pharmaceutical companies, and regulators to happily embark on this new treatment concept. However, based on our understanding of the emergence and selection of anti-malarial drug resistance over the past century, TACT or in more general combination of three or more anti-malarials may exactly be what needs to be implemented to reduce the likelihood of rapid selection of drug resistance (Table 2).

Pharmaceutical companies have been pivotal in the development of new anti-malarials including the most widely used combination therapies artemether-lumefantrine, dihydroartemisinin-piperaquine, artesunateamodiaquine or more recently pyronaridine-artesunate. However, it may be argued that in the past it was mostly academic institutions in collaboration with the World Health Organization driving new therapeutic concepts for treatment of malaria. This includes the shift from anti-malarial monotherapies to ACT, as well as the subsequent global ban of artesunate monotherapy $[4,5,22]$. A decision to recommend a switch from ACT to TACT will be a strategic choice based on the understanding of the history of anti-malarial therapy and evolving evidence from clinical trials. This decision will have to be balanced on the benefit of individual patients in terms of efficacy, tolerability, and safety of first-line anti-malarial drug regimens and the interest of future patients by maximizing the impact of first line treatments on the prevention of drug resistance. Even if direct benefit may not be seen in a single clinical trial or clinical development programmes, it should be a conscious decision to embark on this treatment paradigm. This holds true for TACT as well as for other novel anti-malarial drugs of new classes currently in the clinical development pipeline that may be used in anti-malarial combinations of two or more drugs.

In summary, clinical researchers, funders, regulators and the public health community have to wholeheartedly accept a difficult situation and need to be open to unconventional choices. Current clinical development programmes for TACT will most likely not be able to prove superior efficacy or equal tolerability compared to currently used ACT. Similarly, it will prove difficult to demonstrate their potential to prevent the development of drug resistance in individual clinical trials. Based on the lessons learned over the past century and the analogies to antimicrobial drug resistance, TB and HIV, it may still be the right time and the right place to continue the development of TACT while accepting that convincing evidence for or against their use will have to be assessed over the long run. 


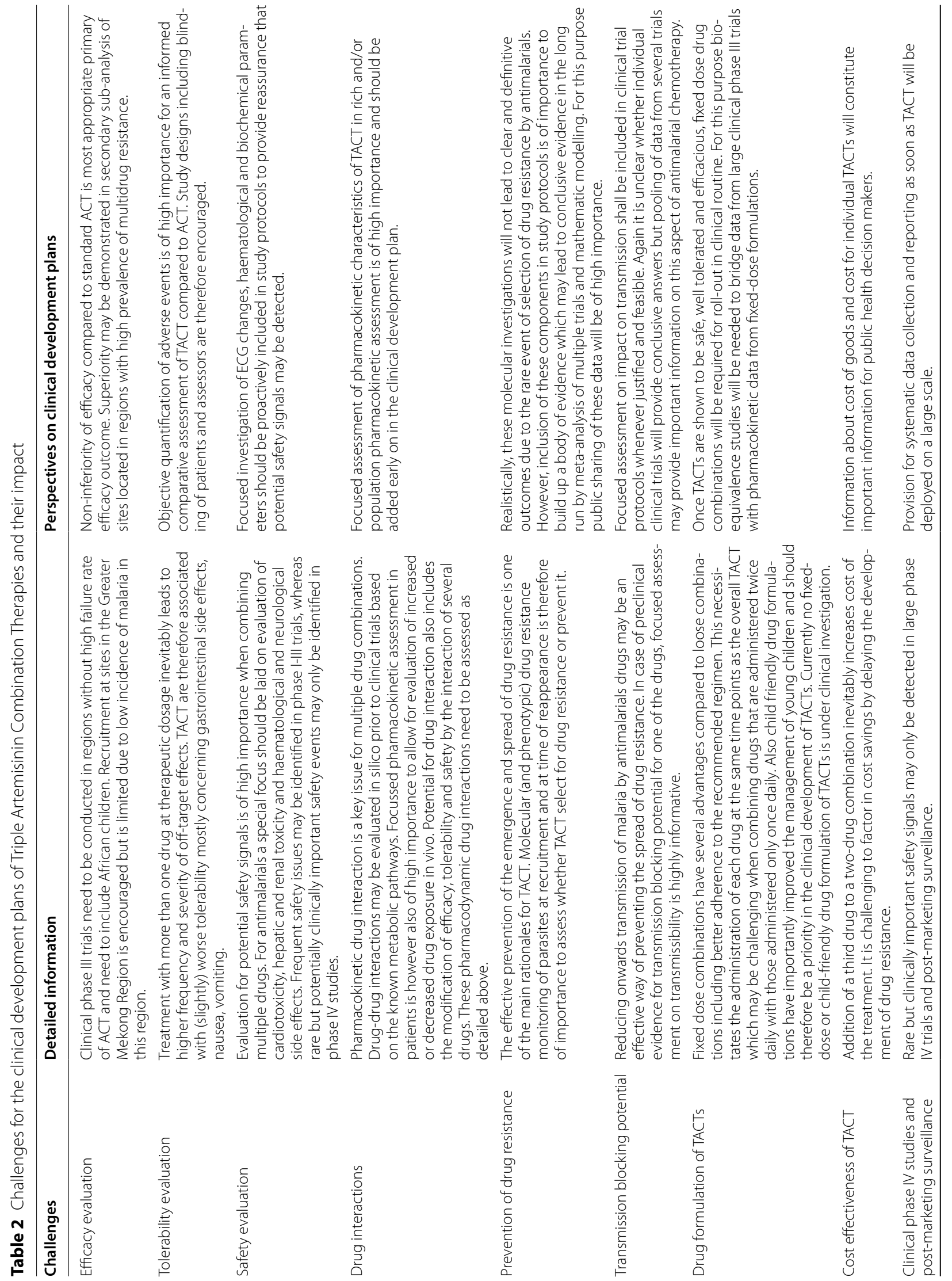




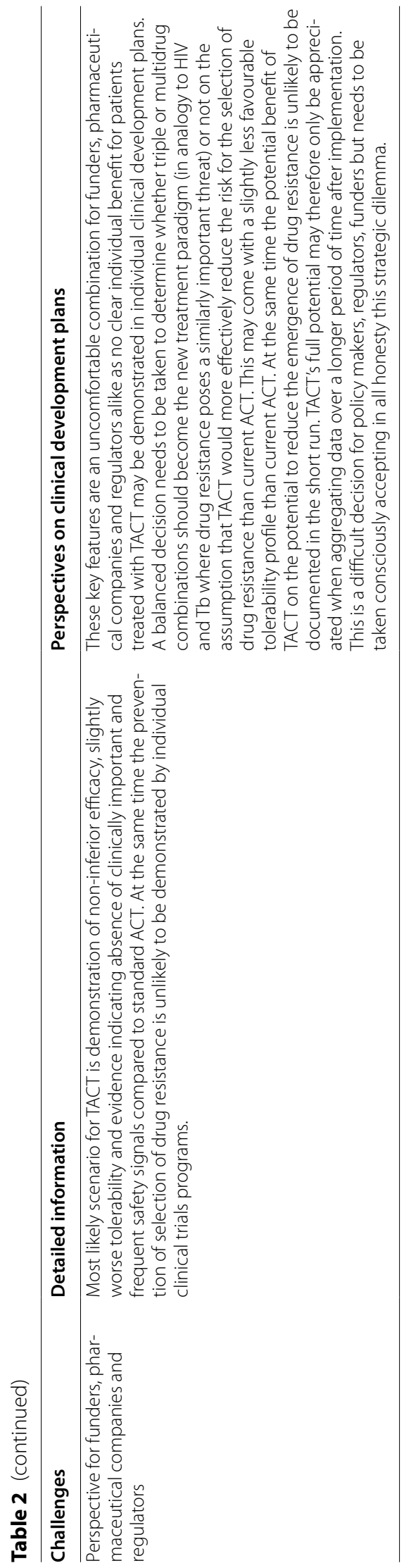




\begin{abstract}
Acknowledgements
ASAAP consortium: The ASAAP consortium is an EDCTP funded consortium of European and African research institutions evaluating the combination of artemether-lumefantrine with atovaquone-proguanil in a randomized placebo controlled clinical phase III trial in five African countries for the treatment of uncomplicated malaria in young children. More information about the ASAAP consortium can be found at: https://asaap-malaria.org/. MULTIMAL consortium: The MULTIMAL consortium is a consortium funded by the German Center for Infection Research to evaluate triple and multidrug combinations for the treatment of uncomplicated malaria in sub-Saharan Africa. The consortium evaluates in an age-deescalation clinical phase II trial the efficacy, safety, tolerability and pharmacokinetics of pyronaridineartesunate combined with atovaquone-proguanil, artesunate-fosmidomycinclindamycin against the standard pyronaridine-artesunate therapy for uncomplicated malaria in Ghana and Gabon. More information about the MULTIMAL consortium can be obtained at https://pactr.samrc.ac.za/TrialDispl ay.aspx?Triall $\mathrm{D}=9740$.
\end{abstract}

\section{Authors' contributions}

MR conceived the opinion paper. All authors and listed members of the ASAAP and MULTIMAL consortia have contributed to the writing and critical revision of the manuscript. All authors have read and approved the final manucsript.

\section{Funding}

Open Access funding enabled and organized by Projekt DEAL.

\section{Availability of data and materials}

Not applicable.

\section{Declarations}

\section{Ethics approval and consent to participate}

Not applicable.

\section{Consent for publication}

Not applicable.

\section{Competing interests}

The authors are engaged in the clinical development of TACTs and multi-drug antimalaria combinations. None of the authors has a financial conflict of interest.

\section{Author details}

${ }^{1}$ ISGlobal, Hospital Clínic - Universitat de Barcelona, Barcelona, Spain. ${ }^{2}$ Centro de Investigação em Saúde de Manhiça (CISM), Maputo, Mozambique. ${ }^{3}$ ICREA, Passeig de Lluís Companys 23, 08010 Barcelona, Spain. ${ }^{4}$ Pediatrics Department, Hospital Sant Joan de Déu, Universitat de Barcelona, Esplugues, Barcelona, Spain. ${ }^{5}$ Consorcio de Investigación Biomédica en Red de Epidemiología y Salud Pública (CIBERESP), Madrid, Spain. ${ }^{6}$ Kumasi Center for Collaborative Research, Kumasi, Ghana. ${ }^{7}$ Bernhard Nocht Institute for Tropical Medicine, Hamburg, Germany. ${ }^{8}$ German Center for Infection Research, Partner Site Hamburg- Lübeck-Borstel-Riems, Hamburg- Lübeck-Borstel-Riems, Germany. ${ }^{9}$ Université de Paris, Centre National de Référence du Paludisme, Hôpital Bichat- Claude Bernard, Assistance Publique des Hôpitaux de Paris, Paris, France. ${ }^{10}$ Centre de Recherches de Lambaréné, Lambaréné, Gabon. ${ }^{11}$ Institute for Tropical Medicine, University of Tübingen, Tübingen, Germany. ${ }^{12}$ Malaria Research and Training Centre (MRTC), Faculty of Pharmacy, Université des Sciences, des Techniques et des Technologies de Bamako (USTTB), Bamako, Mali.

\section{Received: 24 August 2021 Accepted: 6 February 2022}

Published online: 22 February 2022

\section{References}

1. Wernsdorfer WH, McGregor IA. Malaria: principles and practice of malariology. Edinburgh: Churchill Livingstone Publ; 1989.

2. Harinasuta T, Bunnag D, Wernsdorfer WH. A phase II clinical trial of mefloquine in patients with chloroquine-resistant falciparum malaria in Thailand. Bull World Health Organ. 1983;61:299-305.
3. Dixon KE, Williams RG, Pongsupat T, Pitaktong U, Phintuyothin P. A comparative trial of mefloquine and fansidar in the treatment of falciparum malaria: failure of fansidar. Trans R Soc Trop Med Hyg. 1982;76:664-7.

4. Nosten F, Luxemburger C, ter Kuile FO, Woodroow C, Eh JP, Chongsuphajaisiddhi T, et al. Treatment of multidrug-resistant Plasmodium falciparum malaria with 3-day artesunate-mefloquine combination. J Infect Dis. 1994;170:971-7.

5. White NJ, Nosten F, Looareesuwan S, Watkins WM, Marsh K, Snow RW, et al. Averting a malaria disaster. Lancet. 1999;353:1965-7.

6. Trape JF, Pison G, Preziosi MP, Enel C, Desgrées du Loû A, Delaunay V, et al. Impact of chloroquine resistance on malaria mortality. C R Acad Sci III. 1998:321:689-97.

7. Noedl H, Se Y, Schaecher K, Smith BL, Socheat D, Fukuda MM, et al. Evidence of artemisinin-resistant malaria in western Cambodia. N Engl $\mathrm{J}$ Med. 2008;359:2619-20.

8. Menard D, Khim N, Beghain J, Adegnika AA, Shafiul-Alam M, Amodu O, et al. A worldwide map of Plasmodium falciparum K13-propeller polymorphisms. N Engl J Med. 2016;374:2453-64.

9. Leang R, Taylor WR, Bouth DM, Song L, Tarning J, Char MC, et al. Evidence of Plasmodium falciparum malaria multidrug resistance to artemisinin and piperaquine in Western Cambodia: dihydroartemisinin-piperaquine open-label multicenter clinical assessment. Antimicrob Agents Chemother. 2015;59:4719-26.

10. Saunders DL, Vanachayangkul P, Lon C. Dihydroartemisinin-piperaquine failure in Cambodia. N Engl J Med. 2014;371:484-5.

11. van der Pluijm RW, Tripura R, Hoglund RM, Pyae Phyo A, Lek D, Islam AU, et al. Triple artemisinin-based combination therapies versus artemisininbased combination therapies for uncomplicated Plasmodium falciparum malaria: a multicentre, open-label, randomised clinical trial. Lancet. 2020;395:1345-60.

12. van der Pluijm RW, Amaratunga C, Dhorda M, Dondorp AM. Triple artemisinin-based combination therapies for malaria - a new paradigm? Trends Parasitol. 2021;37:15-24.

13. WHO. World Malaria Report 2020. Geneva: World Health Organization; 2020.

14. Uwimana A, Umulisa N, Venkatesan M, Svigel SS, Zhou Z, Munyaneza T, et al. Association of Plasmodium falciparum kelch $13 \mathrm{R} 561 \mathrm{H}$ genotypes with delayed parasite clearance in Rwanda: an open-label, single-arm, multicentre, therapeutic efficacy study. Lancet Infect Dis. 2021;21:1120-8.

15. Hamaluba M, van der Pluijm RW, Weya J, Njuguna P, Ngama M, Kalume P, et al. Arterolane-piperaquine-mefloquine versus arterolane-piperaquine and artemether-lumefantrine in the treatment of uncomplicated Plasmodium falciparum malaria in Kenyan children: a single-centre, open-label, randomised, non-inferiority trial. Lancet Infect Dis. 2021;21:1395-406.

16. Tripura R, Peto TJ, Nguon C, Davoeung C, Mukaka M, Sirithiranont P, et al. A controlled trial of mass drug administration to interrupt transmission of multi drug resistant falciparum malaria in Cambodian villages. Clin Infect Dis. 2018;67:817-26.

17. Ouologuem DT, Kone CO, Fofana B, Sidibe B, Togo AH, Dembele D, et al Differential infectivity of gametocytes after artemisinin-based combination therapy of uncomplicated falciparum malaria. Afr J Lab Med. 2018;7:784

18. Chen PQ, Li GQ, Guo XB, He KR, Fu YX, Fu LC, et al. The infectivity of gametocytes of Plasmodium falciparum from patients treated with artemisinin. Chin Med J (Engl). 1994;107:709-11.

19. Roper C, Pearce R, Bredenkamp B, Gumede J, Drakeley C, Mosha F, et al. Antifolate antimalarial resistance in southeast Africa: a population-based analysis. Lancet. 2003;361:1174-81.

20. Hastings IM. The origins of antimalarial drug resistance. Trends Parasitol. 2004;20:512-8

21. Smith DL, McKenzie FE, Snow RW, Hay SI. Revisiting the basic reproductive number for malaria and its implications for malaria control. PLoS Biol. 2007;5:e42.

22. https://www.who.int/malaria/areas/greater_mekong/assessment_ report-response-to-artemisinin-resistance-gms-2012.pdf. Accessed 19 Sept 2021.

\section{Publisher's Note}

Springer Nature remains neutral with regard to jurisdictional claims in published maps and institutional affiliations. 